\title{
Genetic Change of Varicella-Zoster Virus Propagated in Cell Culture in Non-Natural Conditions
}

\author{
Sang Hoon Yeon, Ji Seon Park, Se Hwan Kang, Chan Hee Lee* \\ Department of Microbiology, Chungbuk National University, Cheongju, Chungbuk 28644, Republic of Korea
}

\section{Corresponding}

Chan Hee Lee, Ph.D.

1 Chungdae-Ro, Seowon-Gu, Cheongju, Chungbuk 28644, Korea

Department of Microbiology, Chungbuk

National University, Republic of Korea

Phone : +82-43-261-2304

Fax : +82-43-273-2451

E-mail : chlee@chungbuk.ac.kr

Received : August 30, 2021

Revised : December 6, 2021

Accepted : December 6, 2021

No potential conflict of interest relevant to this article was reported.

Copyright (C) 2021 Journal of Bacteriology and Virology

(C) This is an Open Access article distributed under the terms of the Creative Commons Attribution Non-Commercial License

(http://creativecommons.org/ license/by-nc/3.0/).
Primary infection of varicella-zoster virus (VZV) causes varicella and often leads to zoster after reactivation from latency. Both varicella and zoster can be prevented by live attenuated vaccines, but the molecular mechanism of attenuation is not clearly understood. In this study, it was attempted to understand mechanism of attenuating mutation in VZV by in vitro propagation in non-natural conditions such as low temperature or non-human cell. Clinical strain YCO2 was subcultured in vitro up to 60 times. Comparison of the genome sequences of YCO2 variants cultured under various conditions identified specific mutations occurred in non-natural conditions. The mutations specific for low temperature culture and non-human cell culture were identified in 8 and 2 positions, respectively. Two vaccine-specific mutations in position 97748 and 106262 were identified during subculture in non-natural conditions. Genetic diversity as measured by genetic polymorphism and Shannon entropy decreased when cultured in guinea pig lung cell culture. The infectivity of YCO2 cultured at low temperature appeared similar to that cultured in natural condition. On the other hands, infectivity decreased significantly when YCO2 was subcultured in non-human cell. Further studies on mutations and genetic diversity of clinical strain cultured in non-natural conditions will help to elucidate the molecular mechanism of VZV attenuation.

Key Words: Varicella-zoster virus, Vaccine, Low temperature culture, Non-human cell culture, Mutation, Genetic diversity

\section{INTRODUCTION}

Varicella-zoster virus (VZV) belongs to the subfamily Alphaherpesvirinae of the Herpesviridae family, and is the smallest and genetically stable among eight human herpes viruses. VZV has a genome of about $125 \mathrm{Kbp}$, linear double-stranded DNA, and encodes 74 open reading frames (ORFs). The first VZV strain whose entire nucleotide sequence was identified is Dumas (NC001348), which is used as a reference in research and analysis of VZV (1).

VZV causes varicella as a primary infection and exists as a latent infection in sensory ganglia. Afterwards, if the immune system is weakened or in the elderly, the virus reactivates and causes herpes zoster $(2,3)$. Aerosolized varicella virion from the lesion enters the upper respiratory tract via droplets and infects $T$ cells (4). Infected T cells migrate to the skin via blood, multiply and cause rashes and blisters, which is called chickenpox. Infectivity is maintained for 3-7 days after the 
rash appears or until the blisters become crusted. Afterwards, when the cellular immunity weakens the virus dormant in the sensory ganglion reactivates and forms a band-shaped herpes on one side of the face or body, which is called herpes zoster. Rash caused by herpes zoster usually disappears within 2-4 weeks, after which the neuralgia may persist for months to years, which is called postherpetic neuralgia $(5,6)$. Chickenpox occurs predominantly in children and occurs in $50 \%$ of children before the age of 5 , and is experienced in $90 \%$ by the time they are 12 years of age (7). In addition, it is highly contagious, and is often transmitted through family contact. In Korea, chickenpox was designated as a legal communicable disease in 2005 , and the chickenpox vaccine was included in the basic vaccination.

Varicella and zoster are currently prevented by live attenuated vaccines. A wild strain was isolated from a 3-year-old boy who had chickenpox in Japan in 1974, and attenuated through subculture to develop a vaccine called vOka (8). The varicella vaccine was commercialized in Japan from 1988. Afterwards, modified formulation based on vOka was developed by Merck and GlaxoSmithKline in the United States (9, 10), and basic inoculation was performed from 1995 (11). In Korea, Green Cross has developed a chickenpox vaccine called SuduVax using the virus isolated from a 33-month-old boy with chickenpox in Seoul (12). SuduVax was sold in Korea in 1994 and commercialized internationally in 1998.

Attenuated varicella vaccine produced through subculture is used throughout the world, but the mechanism of vaccine attenuation has not been precisely elucidated. Characteristics of the attenuated vaccine development process include non-natural culture conditions such as low temperature culture and culturing of the virus in guinea pig cells. In this study, we tried to understand the attenuation mechanism by comparing clinical strains subcultured in non-natural culture conditions at low temperature and in guinea pig cells with those cultured in natural conditions.

\section{MATERIALS AND METHODS}

\section{Virus culture}

In a previous study, YC02, a clinical strain of VZV, was isolated from a 3-year-old boy who developed chickenpox (13). YCO2 was grown in human foreskin fibroblast (HFF, passage 16-22) cells at $37^{\circ} \mathrm{C}$ and $5 \% \mathrm{CO}_{2}$. In order to replicate at low temperature, YCO2 at passage 17 (p17) was subcultured in HFF cells at $34^{\circ} \mathrm{C}$ until passage 60 . For replication of YC02 in guinea pig cells, YCO2 cultured at $34^{\circ} \mathrm{C}$ until passage 30 (p30 (34)) was transferred to $37^{\circ} \mathrm{C}$. After 4 passage at $37^{\circ} \mathrm{C}$, virus was inoculated into guinea pig lung cells (GPLC, ATCC No. CCL-158, passage 34-40) and cultured at $37^{\circ} \mathrm{C}$. Serial passaging scheme of VZV YCO2 in different culture conditions is summarized in Fig. 1.

Dulbecco's modified Eagle medium (DMEM, Gibco, Thermo Fisher Scientific, Waltham, MA, USA) and F-12 nutrient mixture medium (Gibco) containing 10\% fetal bovine serum (FBS, Gibco), 100 mg/ml streptomycin (Sigma-Aldrich, St. Louis, MO, USA) and $100 \mathrm{U} / \mathrm{ml}$ penicillin (Sigma-Aldrich) were used for HFF and GPLC, respectively. Cells grown as a monolayer in a

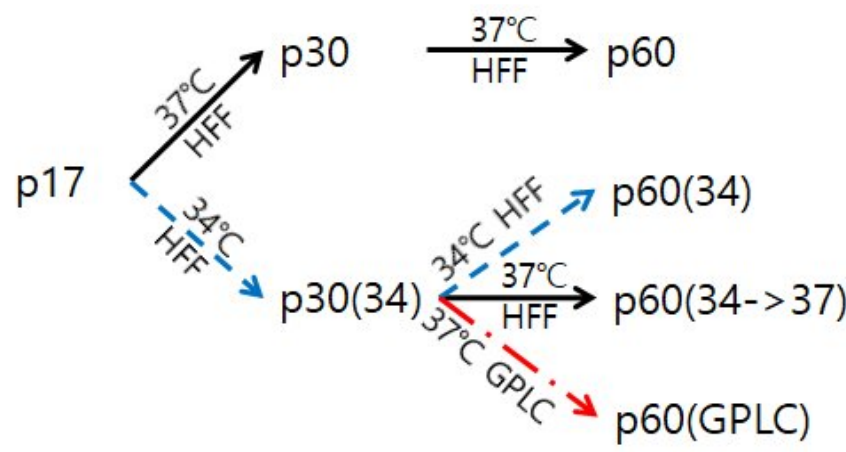

Fig. 1. Overview of serial passaging of VZV. HFF: human foreskin fibroblast; GPLC: guinea pig lung cell. 
cell culture flask was inoculated by the virus diluted in a ratio of $1: 8$. After $1 \mathrm{hr}$ adsorption at $37^{\circ} \mathrm{C}$ in $5 \% \mathrm{CO}_{2}$, virus inoculum was removed and a medium containing $2 \%$ FBS was added. The virus was subcultured until passage 60 .

\section{Virus harvest and concentration}

Virus samples were harvested at every 5 passages for infectivity assay or whole genome sequencing. When the virus-infected cells showed a cytopathic effect (CPE) of $80 \%$ or more, the medium was removed, washed once with phosphate-buffered saline (PBS, pH 7.4), and trypsin-ethylenediaminetetraacetic acid (EDTA, Sigma-Aldrich) was added. After incubation at $37^{\circ} \mathrm{C}$ for $3 \mathrm{~min}$, the cells were suspended in serum-free DMEM, and centrifuged at $800 \times \mathrm{xg}$ for $10 \mathrm{~min}$. The cell pellet was suspended in a mixture of $90 \%$ cell-freezer (Genenmed, Seoul, Korea) and $10 \%$ dimethylsulfoxide (Sigma-Aldrich), and stored in liquid nitrogen until further assay. Even if CPE did not appear in the virus-infected GPLC, the virus was harvested in the same way 3-4 days after virus inoculation, and F-12 medium was used instead of DMEM.

Since virus samples obtained from infected cells contain large amounts of cellular debris in addition to the viral genome, it was necessary to remove cellular impurities as much as possible and to concentrate the virus for genome sequencing. For this, the freezing \& thawing process was repeated 3 times followed by 12 cycles of sonication (Sonics, VCX130, Newton, CT, USA) at an amplitude of $20 \%$ for 10 seconds. The sonicated samples were centrifuged at 8,000 rpm for 5 min at $4^{\circ} \mathrm{C}$. For virus concentration, $0.8 \mathrm{~g} / \mathrm{ml}$ ammonium sulfate $\left(\left(\mathrm{NH}_{4}\right)_{2} \mathrm{SO}_{4}\right)$ was mixed 1:1 with the supernatant, stirred for 4 hrs, and centrifuged at $10,000 \mathrm{xg}, 4^{\circ} \mathrm{C}$ for $30 \mathrm{~min}$. The precipitate was suspended in $200 \mu \mathrm{l}$ of serum-free medium.

\section{DNA extraction and next generation sequencing (NGS)}

DNA was extracted from the concentrated virus using Exgene ${ }^{\mathrm{TM}}$ Cell SV mini kit (GeneAll Biotechnology, Seoul, Korea). To estimate the amount of viral DNA and cellular DNA, q-PCR was performed with IQ SYBR Green SuperMix (BIO-RAD, Hercules, CA, USA). Using previously known references, two pairs of primers capable of detecting cellular DNA and VZV DNA were used. To check the amount of cellular DNA, a primer that amplifies the glyceraldehyde-3-phosphate dehydrogenase (GAPDH) site was used, and to check the amount of viral DNA in VZV, a primer that amplifies the ORF4 site was used $(14,15)$. PCR was repeated 45 times under the conditions of initial denaturation at $94^{\circ} \mathrm{C}$ for 4 min, denaturation at $94^{\circ} \mathrm{C}$ for 30 seconds, annealing at $48.9^{\circ} \mathrm{C}$ for 30 seconds, and extension at $72^{\circ} \mathrm{C}$ for 30 seconds.

Whole genome sequence of the extracted viral DNA was determined with Illumina's Hi-Seq 2500 NGS system (San Diego, CA, USA), and this process was carried out by Theragen Etex (Suwon, Korea). Illumina TruSeq DNA Sample Preparation kit (v2) was used to prepare the DNA library, and the prepared library was sequenced in the Hi-Seq method. After quality check and data trimming with Illumina version 1.8, we obtained mapped reads between 244,242 and 3,449,279 and read depth between 196 and 2,768. For reads with guaranteed quality, mapping assembly was performed using the clinical strain YCO2 (MF004348) as a reference sequence. As a result of mapping assemble, one complete nucleotide sequence was obtained.

\section{Genomic analysis}

Information on open reading frame (ORF) and non-coding region (NCR) of each YCO2 variant was explored using local blast search against VZV reference strain Dumas (NC001348). And the ORF check function of CLC Sequence Viewer (version 6.4, http://www.clcbio.com), and ORF Finder provided by NCBI (http://www.ncbi.nlm.nih.gov/) programs were utilized to check whether the searched ORF location was correct and whether there was an additional ORF location. BioEdit Sequence Alignment Editor (version 7.0.9.0, http://www.mbio.ncsu.edu/bioedit) was used to check and confirm whether the ORF has an intact start codon and stop codon in the actual nucleotide sequence. 
For mutation and genetic polymorphism analysis, it was necessary to align the YCO2 base sequence with respect to the standard virus strain. The virus strain used as a standard was Dumas (NC001348), the first to determine the entire nucleotide sequence among the VZV, and the entire nucleotide sequence was used. The entire base sequence and amino acid sequence for each passage were read with BioEdit and aligned using Clustal W2 (version 2.1).

\section{Genetic polymorphism analysis}

Genetic polymorphism refers to having two or more bases together at any same nucleotide position in a genome. Information on the number of $A, C, G$, and $T$ bases for each position of the nucleotide sequence was obtained from the NGS analysis of the YCO2 variants. Among the bases mapped for each position, the base that occupies the largest proportion is defined as the major base, and constitutes the representative or consensus nucleotide sequence. The base that occupies the second largest proportion is determined as a minor base. The Genetic Polymorphism Analyzer (GPA) program (https://github.com/lis123kr/GPA) developed in our laboratory was used for analysis. This program compares the ratio of minor bases between sequences, finds genetic polymorphisms, and shows the results of analysis in various forms. In this study only the genetically polymorphic site (GPS) having a minor base ratio of $5 \%$ or more and a read-depth of 35 or more was analyzed (16).

\section{Virus infectivity and growth kinetics}

In order to measure the infectivity of the virus, plaque assay was performed every 5 passages during subculture. The harvested virus sample (see above) was serially 10-fold diluted in serum-free DMEM. In a 6-well plate in which HFF was grown as a monolayer, $200 \mu \mathrm{l}$ per well of the virus dilution was inoculated and adsorbed for $1 \mathrm{hr}$ with gentle shaking every 15 min. Thereafter, DMEM containing 2\% FBS was added and incubated. After 7 days, the medium was removed, washed once with PBS ( $\mathrm{pH} 7.4$ ), $10 \%$ formalin (in $0.85 \%$ saline) was added, and the cells were fixed overnight. The next day, after removing the formalin and staining the fixed cells using crystal violet $(0.03 \% \mathrm{w} / \mathrm{v}$ in $10 \%$ ethanol, Sigma-Aldrich), the plaques were counted under a dissecting microscope with a 10X magnification.

Growth kinetics of YCO2 variants was investigated by infecting HFF grown in $60 \mathrm{~mm}$ dishes with virus samples at multiplicity of infection of 0.02 plaque forming units (pfu)/cell. After one hr adsorption at $37^{\circ} \mathrm{C}$, virus inoculum was removed and the infected cultures were fed with DMEM containing $2 \%$ FBS. At 1, 2, 3, 4 and 5 days post infection (d p.i.), infected cells were harvested by trypsinization and centrifuged at $800 \mathrm{xg}$. Infected cell pellets were resuspended with freezing media and stored in liquid nitrogen until plaque assay.

\section{RESULTS}

\section{Mutations that occurred during propagation at low temperature}

In order to find out what genetic variations occurred when the virus was propagated in non-natural conditions, the VZV clinical isolate $\mathrm{YCO} 2$ was subcultured at low temperature $\left(34^{\circ} \mathrm{C}\right)$ and non-human cells (GPLC) (Fig. 1). At the 30th passage (p30) and 60th passage (p60), the entire genome sequence of each variant was determined and compared with that of p17 before propagation in non-natural conditions. A case in which sequences with different bases are found in one or more variants is called single nucleotide polymorphism (SNP), and in this study, it was found in a total of 41 nucleotide positions (Table 1).

To find out what mutations occurred in the low-temperature culture, the nucleotide sequence of YC02 p17 before low-temperature culture was compared with those of p30 (34) and p60 (34) that were harvested at p30 and p60 after 
starting to be cultured at $34^{\circ} \mathrm{C}$ from p18. In p30 (34), mutations were observed in two positions, 87656 and 91239 , and were not observed in any other culture conditions, so it can be said that the mutation occurred specifically during the process of the low-temperature culture (Table 1, green background). Both of these are $T$ to $C$ substitution mutations. Since they are $\mathrm{T}$ in $\mathrm{p} 60$ (34), it seems that a back mutation occurred during subculture of $\mathrm{p} 30$ (34) to p60 (34) at $34^{\circ} \mathrm{C}$.

Table 1. Mutations found in VZV strain YCO2 subcultured in non-natural conditions

\begin{tabular}{|c|c|c|c|c|c|c|c|c|c|c|c|}
\hline & Nucleotide & \multicolumn{2}{|c|}{ Change in } & \multirow{2}{*}{$\begin{array}{c}\text { Substitution } \\
\text { type }\end{array}$} & \multicolumn{7}{|c|}{ YCO2 strains } \\
\hline ORF & position & nucleotide & amino acid & & $\mathrm{p} 17$ & p30 & p30 (34) & $\mathrm{p} 60$ & p60 (34) & $\mathrm{p} 60(34-37)$ & p60 (GP) \\
\hline 2 & 001337 & $A->G$ & V51 & Synonymous & A & A & A & A & G & G & G \\
\hline $3^{\prime}$ & 002314 & $T->C$ & $\mathrm{H} 45 \mathrm{R}$ & Non Synonymous & $\mathrm{T}$ & $\mathrm{T}$ & $\mathrm{T}$ & $\mathrm{T}$ & C & C & $\mathrm{T}$ \\
\hline $3 / 4$ & 002676 & $T->G$ & & $\mathrm{~N} / \mathrm{A}$ & $\mathrm{T}$ & $\mathrm{T}$ & $\mathrm{T}$ & $\mathrm{T}$ & G & $\mathrm{T}$ & $\mathrm{T}$ \\
\hline $5^{\prime}$ & 004440 & $A->G$ & L279 & Synonymous & $A$ & $A$ & A & $A$ & G & G & A \\
\hline $6^{\prime}$ & 005596 & $A->G$ & Y994 & Synonymous & A & A & A & G & G & G & G \\
\hline 7 & 009288 & $T->C$ & S228P & Non Synonymous & $\mathrm{T}$ & $\mathrm{T}$ & $\mathrm{T}$ & $\mathrm{T}$ & C & C & $\mathrm{T}$ \\
\hline 11 & 013667 & $T->C$ & $\mathrm{H} 26$ & Synonymous & $\mathrm{T}$ & $\mathrm{T}$ & $\mathrm{T}$ & $\mathrm{T}$ & C & C & $\mathrm{T}$ \\
\hline $14^{\prime}$ & 020829 & $C->A$ & K95N & Non Synonymous & $C$ & $C$ & C & $C$ & A & $C$ & C \\
\hline $14^{\prime}$ & 020963 & $A->T$ & S51T & Non Synonymous & $A$ & $A$ & A & $A$ & $\mathrm{~T}$ & $\mathrm{~T}$ & A \\
\hline $18^{\prime}$ & 026470 & $->A$ & Early stop & & - & - & - & - & - & - & A \\
\hline 22 & 041458 & $C->T$ & A2459V & Non Synonymous & $C$ & $C$ & C & $C$ & $\mathrm{~T}$ & $\mathrm{~T}$ & C \\
\hline 22 & 041459 & $G->C$ & A2459V & Non Synonymous & G & G & G & G & C & C & G \\
\hline $34^{\prime}$ & 063539 & $T->C$ & I124M & Non Synonymous & $\mathrm{T}$ & $\mathrm{T}$ & $\mathrm{T}$ & $\mathrm{T}$ & C & C & C \\
\hline 36 & 065446 & $A->G$ & I214V & Non Synonymous & A & A & A & A & G & A & A \\
\hline 36 & 065448 & $A->G$ & I214V & Non Synonymous & $A$ & $A$ & A & $A$ & G & A & A \\
\hline 37 & 066247 & $T->C$ & F58 & Synonymous & $\mathrm{T}$ & $\mathrm{T}$ & $\mathrm{T}$ & $\mathrm{T}$ & C & C & $\mathrm{T}$ \\
\hline 44 & 080436 & $A->G$ & $\mathrm{~K} 26 \mathrm{R}$ & Non Synonymous & $A$ & A & A & $A$ & G & G & A \\
\hline $50^{\prime}$ & 087656 & $T->C$ & C76Y & Non Synonymous & $\mathrm{T}$ & $\mathrm{T}$ & C & $\mathrm{T}$ & $\mathrm{T}$ & $\mathrm{T}$ & $\mathrm{T}$ \\
\hline 51 & 088867 & $T->C$ & V329 & Synonymous & $\mathrm{T}$ & $T$ & T & $\mathrm{T}$ & C & C & $\mathrm{T}$ \\
\hline 52 & 091239 & $T->C$ & S249 & Synonymous & $\mathrm{T}$ & $\mathrm{T}$ & C & $\mathrm{T}$ & $\mathrm{T}$ & $\mathrm{T}$ & $T$ \\
\hline $54^{\prime}$ & 094135 & $C->T$ & S617K & Non Synonymous & $C$ & $C$ & C & $C$ & C & C & $\mathrm{T}$ \\
\hline 55 & 097525 & $A->G$ & T510 & Synonymous & $A$ & A & A & A & G & G & A \\
\hline 55 & 097748 & G->A & A585T & Non Synonymous & G & G & G & G & A & G & A \\
\hline $59^{\prime}$ & 100653 & $T->C$ & P189 & Synonymous & $\mathrm{T}$ & $\mathrm{T}$ & $\mathrm{T}$ & $\mathrm{T}$ & C & C & $\mathrm{T}$ \\
\hline $61 / 62$ & 104529 & $T->C$ & & N/A & $\mathrm{T}$ & $\mathrm{T}$ & $\mathrm{T}$ & $\mathrm{T}$ & C & $\mathrm{T}$ & $\mathrm{T}$ \\
\hline $61 / 62$ & 105009 & $C->G$ & & $\mathrm{~N} / \mathrm{A}$ & $C$ & $C$ & C & $C$ & G & G & C \\
\hline $61 / 62$ & 105054 & G->- & & $\mathrm{N} / \mathrm{A}$ & G & G & G & G & - & G & G \\
\hline $62^{\prime}$ & 105283 & $A->G$ & L1284S & Non Synonymous & A & A & A & G & G & G & G \\
\hline $62^{\prime}$ & 105413 & $T->C$ & S1241G & Non Synonymous & $\mathrm{T}$ & $C$ & C & $C$ & C & C & C \\
\hline $62^{\prime}$ & 105633 & $A->G$ & L1167 & Synonymous & $A$ & $A$ & A & G & G & G & G \\
\hline $62^{\prime}$ & 106052 & $A->G$ & L1028 & Synonymous & A & A & A & G & G & G & A \\
\hline $62^{\prime}$ & 106262 & $T->C$ & R958G & Non Synonymous & $\mathrm{T}$ & $\mathrm{T}$ & $\mathrm{T}$ & $\mathrm{T}$ & C & C & $\mathrm{T}$ \\
\hline $62^{\prime}$ & 106332 & $T->C$ & L934 & Synonymous & $\mathrm{T}$ & $\mathrm{T}$ & $\mathrm{T}$ & $C$ & C & C & C \\
\hline $62^{\prime}$ & 107045 & $T->C$ & K697E & Non Synonymous & $\mathrm{T}$ & $\mathrm{T}$ & $\mathrm{T}$ & $C$ & C & C & $\mathrm{T}$ \\
\hline $62^{\prime}$ & 107070 & $T->C$ & P688 & Synonymous & $\mathrm{T}$ & $\mathrm{T}$ & $\mathrm{T}$ & $\mathrm{T}$ & C & C & $\mathrm{T}$ \\
\hline $62^{\prime}$ & 107371 & $A->G$ & M588A & Non Synonymous & $A$ & A & A & $A$ & G & G & A \\
\hline $62^{\prime}$ & 107372 & $T->C$ & M588A & Non Synonymous & $\mathrm{T}$ & $\mathrm{T}$ & $\mathrm{T}$ & $\mathrm{T}$ & C & C & $\mathrm{T}$ \\
\hline $62^{\prime}$ & 108345 & $A->G$ & G263 & Synonymous & A & A & A & G & G & G & A \\
\hline $62 / 63$ & 109253 & $C->-$ & & $\mathrm{N} / \mathrm{A}$ & $C$ & $C$ & - & - & - & - & C \\
\hline $62 / 63$ & 110195 & $C->A$ & & $\mathrm{~N} / \mathrm{A}$ & $C$ & A & C & A & A & A & C \\
\hline 63 & 110755 & $A->G$ & R59G & Non Synonymous & A & A & A & G & G & G & G \\
\hline
\end{tabular}


(A)

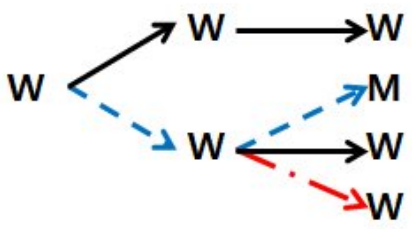

(C)

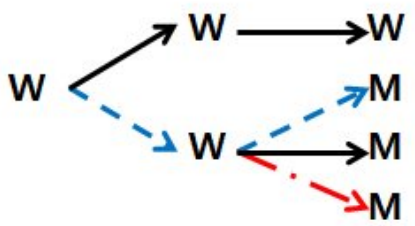

(B)

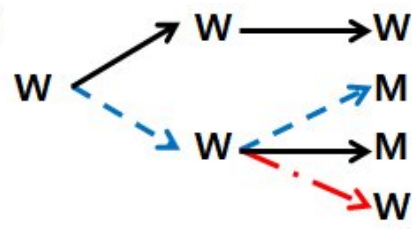

(D)

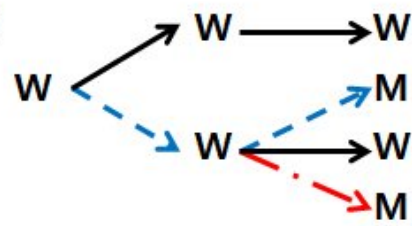

Fig. 2. Four types of mutations found in three lineages of p60 subcultures from p30 (34). (A), mutation was detected only in p60 (34), found at 6 positions: 2676, 20829, 65446, 65448, 104529, 105054. (B), mutations were detected both in p60 (34) and p60 (34->37), found at 17 positions: 2314, 4440, 9288, 13667, 20963, 41458, 41459, 66247, 80436, 88867, 97525, 100653, 105009, 106262, 107070, 107371, 107372. (C), mutations were detected in all three p60's, found at 2 positions:1337, 63539. (D), detected in p60 (34) and p60 (GPLC), found only at 1 position: 97748.

In p60 (34), mutations were observed in 37 nucleotide positions (Table 1, red letter). Among them, 26 mutations were considered to be related to low-temperature culture since mutation was not observed in p60 cultured in natural conditions. They can be further divided into 4 types (Fig. 2). In the first type, mutation was observed only in p60 (34) (Fig. 2A), and were found in 6 nucleotide positions (Table 1, yellow background). It was found in one site in ORF14 and two sites in ORF36. Two mutations in ORF36 occurred at the first and third positions of the same codon, resulting in $1214 \mathrm{~V}$ nonsynonymous substitution. The other three were found in NCR. These first types of mutations can be considered low temperature-specific mutations, occurred during propagation of p30 (34) to p60 (34) at $34^{\circ} \mathrm{C}$. In the second type, same mutation was also observed in p60 (34->37) (Fig. 2B), which was found in 17 sites. In the third type, two mutations in nucleotide positions 1337 and 63539 were observed in all three p60 variants subcultured from p30 (34) (Fig. 2C). The fourth type is observed only in p60 (34) and p60(GPLC), which are non-natural culture conditions (Fig. 2D). Since it does not appear in $p 60$ (34->37), it is thought to be a mutation that occurred under non-natural conditions.

\section{Mutations that occurred during propagation in non-human cell culture}

In p60 (GPLC), 11 nucleotide positions different from p30 (34) were observed (Table 1, green letter). There were two mutations observed only in p60 (GPLC) (Table 1, blue background). Early termination of ORF18 is expected by insertion of A at position 26470. C to T substitution at position 94135 resulted in an S617K nonsynonymous mutation in ORF54. Substitution mutations in the other 9 positions were not considered to be GPLC-specific mutations since mutations were observed in other culture conditions also.

\section{Mutation at vaccine-specific sites}

In our previous study on VZV vaccine, 24 vaccine-specific sites were suggested (13). Among them, the mutations that occurred in the non-natural conditions were found in two nucleotide positions in this study. A585T nonsynonymous mutation occurred in ORF55 due to $G$ to A substitution at position 97748 . Since the mutation in 97748 appeared both in p60 (34) and p60 (GPLC) and not in other culture conditions, it is believed that the mutation occurred during the process of subculture of p30 (34) under non-natural conditions such as low temperature $\left(34^{\circ} \mathrm{C}\right.$ ) or non-human cells (Fig. 2D). $\mathrm{T}$ to C substitution at position 106262 causes the R958G nonsynonymous mutation in ORF62. This mutation was observed in p60 (34) and p60 (34->37) but not in p60 (GPLC). Thus, this mutation is considered to occur during the process of 
Table 2. Genetic polymorphism in VZV strain YCO2 subcultured in non-natural conditions

\begin{tabular}{cccc}
\hline Strain & No. GPS & Average MAF & Hs \\
\hline p17 & 28 & 0.205 & 3.04 \\
p30 & 36 & 0.182 & 3.31 \\
p30 (34) & 31 & 0.201 & 3.10 \\
p60 & 110 & 0.170 & 4.48 \\
p60 (34) & 93 & 0.241 & 4.32 \\
p60 (34 $\rightarrow 37)$ & 101 & 0.229 & 4.38 \\
p60 (GP) & 35 & 0.170 & 3.29 \\
\hline
\end{tabular}

GPS: genetically polymorphic sites

MAF: minor allele frequency

Hs: Shannon entropy

subculture of p30 (34) in HFF, a natural cell regardless of the culture temperature (Fig. 2B). Similar mutations were also found at 16 positions other than position 106262.

\section{Changes in genetic diversity}

Genetic diversity can be expressed in many ways, and genetic polymorphism is one of them. Genetic polymorphism refers to co-existence of two or more bases at a frequency of $5 \%$ or more at any one position on the genome. We examined how genetic polymorphism changed when VZV YCO2 was propagated in non-natural conditions. The site on the genome where genetic polymorphism appears is called a genetically polymorphic site (GPS). The number of GPS increased as VZV was subcultured in natural conditions (Table 2). When subcultured at low temperature, the number of GPS decreased by about $15 \%$ compared to when subcultured at normal temperature, while the minor allele frequency (MAF) in GPS slightly increased. On the other hand, when subcultured in GPLC, the number of GPS was significantly reduced compared to when cultured in natural conditions or at low temperature (Table 2).

Shannon entropy (Hs), another indicator of genetic diversity, is calculated as the sum of the MAF of each GPS divided by Ln (MAF) (17). Hs also increased with subculture in natural conditions. In the case of subculture at low temperature, the Hs was slightly lower than subculture at normal temperature (Table 2). On the other hand, in p60 (GPLC), Hs was significantly reduced. Therefore it is thought that genetic diversity is reduced when YC02 was propagated in GPLC.

\section{Infectivity of VZV YCO2 propagated in non-natural conditions}

In order to examine whether the infectivity of VZV subcultured in non-natural conditions, VZV strain YCO2 cultured in various conditions as shown in Fig. 1 were harvested every 5 passages from p15 to p60, and infectivity was evaluated by plaque assay. There was no significant difference in virus infectivity when cultured at low temperature compared to when cultured at normal temperature (Fig. 3). On the other hand, when cultured in GPLC, the infectivity decreased sharply, and it appeared that there was almost no infectivity between $\mathrm{p} 40$ and p45, and infectivity were gradually recovered thereafter.

The proliferative ability of viruses subcultured up to passage 60 in various culture conditions was investigated using growth kinetics assay in HFF cells. The proliferation of p60 cultured in natural conditions was the highest, and the proliferation of p60 (GPLC) cultured in guinea pig cells was the lowest (Fig. 4). The proliferation of p60 (34) cultured at a low temperature is slightly lower than that of p60. The difference in proliferative ability between p60 and p60 (34) increased on the 4th and 5th days of infection. The proliferative ability of p60 (34->37) was intermediate between that of p60 and p60 (34). 


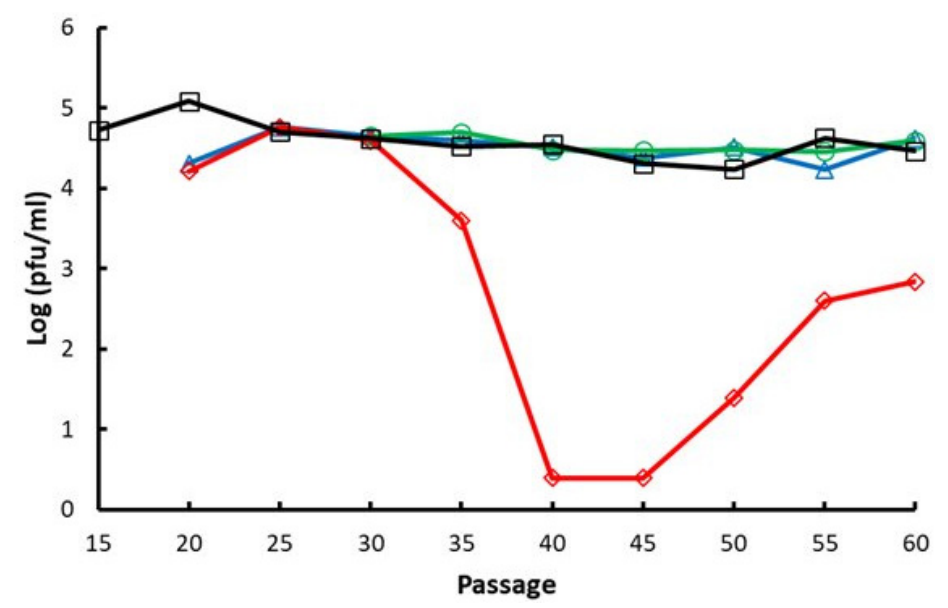

Fig. 3. Changes in the infectious virus titer with propagation of VZV in natural and non-natural conditions in vitro. The VZV strain YCO2 was propagated in HFF and GPLC culture to up to 60 passages. At every 5 passage, the infectious titer was determined by plaque assay. pfu, plaque-forming units. (- $\square-)$ HFF at $37^{\circ} \mathrm{C},\left(-\mathrm{O}^{-}\right) \mathrm{HFF}$ at $34^{\circ} \mathrm{C},(-\triangle-) \mathrm{HFF}$ at $34^{\circ} \mathrm{C}$ and shifted to $37^{\circ} \mathrm{C},(-\diamond-) \mathrm{GPLC}$ at $37^{\circ} \mathrm{C}$.

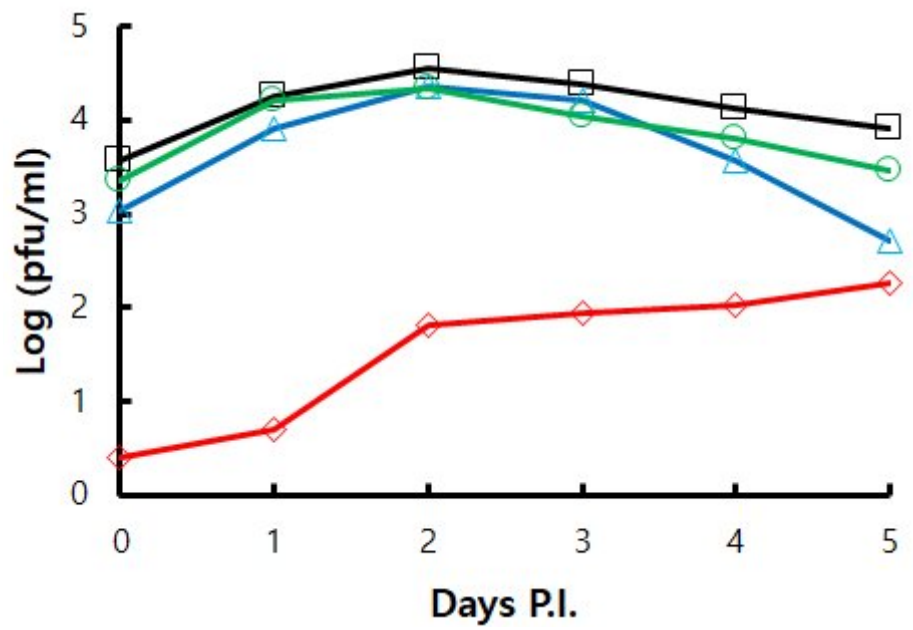

Fig. 4. Growth kinetics of VZV YCO2 subcultured up to passage 60 in various culture conditions. Samples were harvested every day until 5 days post infection (Days P.I). Virus infectivity was determined by plaque assay. (- $\square-)$ HFF at $37^{\circ} \mathrm{C},\left(-\bigcirc^{-}\right) \mathrm{HFF}$ at $34^{\circ} \mathrm{C},\left(-\triangle^{-}\right) \mathrm{HFF}$ at $34^{\circ} \mathrm{C}$ and shifted to $37^{\circ} \mathrm{C},(-\diamond-) \mathrm{GPLC}$ at $37^{\circ} \mathrm{C}$.

\section{Discussion}

Attenuation procedures for varicella vaccine production include propagation of VZV in non-natural conditions such as low temperature and non-human cell culture $(8,9,12)$. Accordingly, we wanted to find out what kind of genetic and biological changes were accompanied during propagation of VZV in non-natural conditions.

One way of non-natural culture conditions is low temperature culture. Since $34^{\circ} \mathrm{C}$ was used in the manufacturing process of the commercial varicella vaccines, this study also tried to find out what kind of genetic change occurred when VZV was propagated at $34^{\circ} \mathrm{C}$. The site where p60 (34) mutation was most observed during low temperature culture was ORF62. ORF62 is a gene encoding a VZV transactivator protein $(18,19)$, and is known as the main mechanism of attenuated vaccines by inhibiting the proliferation and spread of viruses when mutations occur in this region (20). However, the 
mutations found in ORF62 are difficult to consider as low temperature-specific because they are also found in other culture conditions including p60 cultured at normal temperature and p60 (34->37) cultured at a low temperature and shifted to normal temperature.

Low temperature-specific mutations are considered to include two sites that were observed only in p30 (34) and six sites that were observed only in p60 (34). The two sites (positions 87656, 91239) observed only in p30 (34) appeared to undergo a back mutation when continuously cultured in various conditions including normal temperature, low temperature, and non-human cells, so it is difficult to consider it a low temperature-specific mutation. On the other hand, the six mutations observed in p60 (34) did not appear in other culture conditions, thus they can be considered to be low temperature-specific mutations

In previous studies comparing the genome sequences of vaccine and clinical strains, vaccine-specific sequences thought to be related to vaccine attenuation have been suggested $(13,21,22)$. Two of them were identified in this study. Since the $G$ to A mutation at position 97748 appeared in both p60 (34) and p60 (GPLC) but not in other culture conditions, it is thought that the mutation occurred when p30 (34) was subcultured in non-natural conditions. Attenuation of commercial varicella vaccines includes low-temperature culture and non-human cell culture $(9,12,23)$. Therefore, it is thought that the mutation at position 97748 is closely related to propagation in non-natural conditions. The $\mathrm{T}$ to $\mathrm{C}$ mutation at position 106262 was observed in p60 (34) and p60 (34->37), thus it could be considered to be a low temperature-specific mutation. However, same T to C mutation at this position occurred even when YCO2 and another clinical strain, YC01, were cultured up to $\mathrm{p} 110$ in natural culture conditions (24). Therefore, it is controversial whether the $\mathrm{T}$ to $\mathrm{C}$ mutation at position 106262 is a low temperature-specific mutation.

There was no significant difference in the infectivity and proliferation of the virus cultured at low temperature compared to the virus cultured at normal temperature. On the other hand, the infectivity and proliferative power of viruses cultured in GPLC were significantly reduced compared to viruses cultured in natural HFF cells. The infectivity of YCO2 subcultured in non-natural GPLC was decreased but recovered gradually later. It seemed that a very small portion of the virus survived and adapted to GPLC culture. And the adapted virus began to propagate in GPLC. Similar observation was reported previously. Extensive subculture of VZV in HFF cells appeared to induce adaptation to non-natural GPLC culture (24). Genetic diversity showed similar results. Perhaps the low temperature of $34^{\circ} \mathrm{C}$ does not give much stress to virus replication compared to $37^{\circ} \mathrm{C}$, but it seems to be greatly affected when the virus grows in GPLC. In addition to YC02, our laboratory has two more clinical strains, YCO1 and YCO3 (25). Two clinical strains other than YCO2 did not proliferate in GPLC at all, so they were not included in this study.

Many mutations occurred during culture under non-natural conditions such as low temperature and non-human cells. But considering other culture conditions, it is thought that low temperature-specific mutation was found at 6 positions and GPLC-specific mutation at 2 positions. Among them, the mutation that is thought to affect the immune response is the K95N nonsynonymous mutation in ORF14. ORF14 encodes gC. VZV gC is a type 1 transmembrane protein and its ectodomain binds to chemokine to increase migration of leukocytes including tonsillar leukocytes, the main target of VZV primary infection (26). This result seems to be related to the finding that $\mathrm{VZV} \mathrm{gC} \mathrm{is} \mathrm{not} \mathrm{essential} \mathrm{for} \mathrm{in} \mathrm{vitro} \mathrm{replication,} \mathrm{but}$ the replication ability of VZV isolate lacking $\mathrm{gC}$ is reduced in SCID mice (27). Therefore, it will be interesting to further investigate whether the K95N mutation affects the in vitro and in vivo replication of VZV as well as the immune response to VZV infection.

The most common way to attenuate viruses is to culture them in non-natural conditions such as low temperatures and non-human cells (28). Since the varicella vaccine was also produced through the culturing process under such non-natural conditions, it was expected that many of the vaccine-specific mutations could be found through this study. However, only one mutation (position 97748) was found to be associated with a non-natural condition. One of the reason may be that the culture conditions used in this study and the culture conditions when making commercial vaccines were not identical. 
The passage numbers 30 and 60 used in this study were selected with the intention of making them similar to the final passage number of commercial vaccines. However, the actual passage number is 25-38 for Varivax, 35-38 for Varillrix, and 64 for Suduvax, which are somewhat different from passage numbers 30 and 60 used in this study $(12,23)$. Another reason is thought to be that the virus used in the experiment was different. The vaccine strain that produced Suduvax, a Korean varicella vaccine, is called MAV/06, which means that it was the 6th among several clinical strains used in the experiment (12). The other clinical isolates fell short of expectation and failed to produce attenuated vaccine candidates. In this study, clinical strains other than YC02 did not propagate in GPLC at all (data not shown). Even if several clinical strains are passaged with the same protocol, it is difficult to obtain successful attenuation from all clinical strains. In the future, if more clinical strains are cultured in more diverse non-natural conditions, it will be helpful for the study of the attenuation mechanism of the VZV vaccine.

\section{ACKNOWLEDGEMENTS}

This work was conducted during the research year of Chungbuk National University in 2021.

\section{REFERENCES}

1) Davison AJ, Scott JE. The complete DNA sequence of varicella-zoster virus. J Gen Viro/1986;67:1759-816.

2) Gershon AA, Gershon MD. Pathogenesis and current approaches to control of varicella-zoster virus infections. Clin Microbiol Rev 2013:26:728-43.

3) Zerboni L, Sen N, Oliver SL, Arvin AM. Molecular mechanisms of varicella zoster virus pathogenesis. Nat Rev Microbio/ 2014:12:197-210.

4) Arvin AM, Moffat JF, Sommer M, Oliver S, Che $X$, Vleck $S$, et al. Varicella-zoster virus $T$ cell tropism and the pathogenesis of skin infection. Curr Top Microbiol Immuno/2010;342:182-209.

5) Gilden DH, Kleinschmidt-DeMasters BK, Laguardia JJ, Mahalingam R, Cohrs RJ. Neurologic complications of the reactivation of varicella-zoster virus. N Eng/ J Med 2000;342:635-45.

6) Kennedy PGE, Gershon AA. Clinical features of varicella-zoster virus infection. Viruses 2018:10:609.

7) Hall S, Maupin T, Seward J, Jumaan AO, Peterson C, Goldman G, et al. Second varicella infections: are they more common than previously thought? Pediatrics 2002;109:1068-73.

8) Takahashi M, Otsuka T, Okuno Y, Asano Y, Yazaki T. Live vaccine used to prevent the spread of varicella in children in hospital. Lancet 1974;2:1288-90.

9) D’Hondt E, Berge E, Colinet G, Duchene M, Peetermans J. Production and quality control of the Oka-strain live varicella vaccine. Postgrad Med J 1985;61:53-6.

10) Freer G, Pistello M. Varicella-zoster virus infection: natural history, clinical manifestations, immunity and current and future vaccination strategies. New Microbio/ 2018;41:95-105.

11) Gershon AA. The current status of live attenuated varicella vaccine. Arch Viro/ Supp/2001;1-6.

12) Sohn YM, Park CY, Hwang KK, Woo GJ, Park SY. Safety and immunogenicity of live attenuated varicella virus vaccine (MAV/06 strain). J Korean Ped 1994;37:1405-13.

13) Jeon JS, Won $\mathrm{YH}$, Kim IK, Ahn JH, Shin OS, Kim JH, et al. Analysis of single nucleotide polymorphism among 
varicella-zoster virus and identification of vaccine-specific sites. Virology 2016:496:277-86.

14) Cricca M, Morselli-Labate AM, Venturoli S, Ambretti S, Gentilomi GA, Gallinella G, et al. Viral DNA load, physical status and E2/E6 ratio as markers to grade HPV16 positive women for high-grade cervical lesions. Gynecol Oncol 2007:106:549-57.

15) Schmutzhard J, Merete Riedel H, Zweygberg Wirgart B, Grillner L. Detection of herpes simplex virus type 1, herpes simplex virus type 2 and varicella-zoster virus in skin lesions. Comparison of real-time PCR, nested PCR and virus isolation. J Clin Viro/2004;29:120-6.

16) Hedrick PW. Genetics of population. Jones and Bartlett publishers 2010;103-5.

17) Gregori J, Perales C, Rodriguez-Frias F, Esteban Jl, Quer J, Domingo E. Viral quasispecies complexity measures. Virology 2016:493:227-37.

18) Baudoux L, Defechereux P, Schoonbroodt S, Merville MP, Rentier B, Piette J. Mutational analysis of varicella-zoster virus major immediate-early protein IE62. Nucleic Acids Res 1985:23:1341-9.

19) Barrett-Muir W, Hawrami K, Clarke J, Breuer J. Investigation of varicella-zoster virus variation by heteroduplex mobility assay. Arch Viro/ Supp/2001;17-25.

20) Sen N, Sommer M, Che X, White K, Ruyechan WT, Arvin AM. Varicella-zoster virus immediate-early protein 62 blocks interferon regulatory factor 3 (IRF3) phosphorylation at key serine residues: a novel mechanism of IRF3 inhibition among herpesviruses. J Viro/2010;84:9240-53.

21) Gomi Y, Sunamachi H, Mori Y, Nagaike K, Takahashi M, Yamanishi K. Comparison of the complete DNA sequences of the Oka varicella vaccine and its parental virus. J Viro/2002;76:11447-59.

22) Tillieux SL, Halsey WS, Thomas ES, Voycik JJ, Sathe GM, Vassilev V. Complete DNA sequences of two oka strain varicella-zoster virus genomes. J Viro/2008;82:11023-44.

23) Quinlivan M, Breuer J. Clinical and molecular aspects of the live attenuated Oka varicella vaccine. Rev Med Virol 2014:24:254-73

24) Kim SC, Won YH, Park JS, Jeon JS, Ahn JH, Song MJ, et al. Vaccine-type mutations identified in Varicella zoster virus passaged in cell culture. Virus Res 2018;245:62-8.

25) Kim MH, Jeon JS, Kim IK, Park JS, Park H, Shin OS, et al. Characterization and phylogenetic analysis of Varicella-zoster virus strains isolated from Korean patients. J Microbio/2017:55:665-72.

26) González-Motos V, Jürgens C, Ritter B, Kropp KA, Durán V, Larsen O, et al. Varicella zoster virus glycoprotein C increases chemokine-mediated leukocyte migration. PLoS Pathog 2017:13:e1006346.

27) Moffat JF, Zerboni L, Kinchington PR, Grose C, Kaneshima H, Arvin AM. Attenuation of the vaccine Oka strain of varicella-zoster virus and role of glycoprotein $C$ in alphaherpesvirus virulence demonstrated in the SCID-hu mouse. J Virol 1998;72:965-74.

28) Plotkin SA, Orenstein WA, Offit PA. Vaccines, 6th ed. Elsevier, 2013. 